\title{
Maximal determinants of combinatorial matrices
}

\author{
Henning Bruhn Dieter Rautenbach
}

\begin{abstract}
We prove that $\operatorname{det} A \leq 6^{\frac{n}{6}}$ whenever $A \in\{0,1\}^{n \times n}$ contains at most $2 n$ ones. We also prove an upper bound on the determinant of matrices with the $k$-consecutive ones property, a generalisation of the consecutive ones property, where each row is allowed to have up to $k$ blocks of ones. Finally, we prove an upper bound on the determinant of a path-edge incidence matrix in a tree and use that to bound the leaf rank of a graph in terms of its order.
\end{abstract}

\section{Introduction}

There is a rich tradition of bounding the determinants of matrices with all entries 0 or 1 (or -1 and 1). Yet, even very simple questions remain open. For instance:

Problem 1. Given a matrix $A \in\{0,1\}^{n \times n}$ with at most $2 n$ ones, how large can its determinant be?

In fact, Problem 1 is open for any linear number $O(n)$ of ones in $A$, and we consider the case of at most $2 n$ as a simple and restricted representative. Furthermore, this case naturally relates to edge-vertex incidence matrices of graphs, which we exploit below.

We give an answer to this question as well as to questions about the determinants of similarly simple matrices.

The most prominent question in this area is probably Hadamard's maximal determinant problem: Given $A \in\{-1,1\}^{n \times n}$, how large can $\operatorname{det} A$ be? A partial answer lies in Hadamard's inequality [11. For this, let us denote the $i$ th row of a matrix $A \in \mathbb{R}^{n \times n}$ by $A_{i, \cdot}$, and similarly we write $A_{\cdot, j}$ for the $j$ th column. Then

$$
|\operatorname{det}(A)| \leq \prod_{i=1}^{n}\left\|A_{i, \cdot}\right\|_{2} .
$$

If $A$ is a $-1 / 1$-matrix, then (1D) implies $|\operatorname{det}(A)| \leq n^{n / 2}$, and $A$ is a Hadamard matrix of order $n$ if this inequality holds with equality. It is known that a Hadamard matrix of order $n$ can exist only if $n \in\{1,2\} \cup 4 \mathbb{N}$. Paley's conjecture [17] states that for all these orders, Hadamard matrices actually do exist.

Coming back to Problem 1, with the inequality of the arithmetic and geometric mean, it is not hard to derive from (1) that $\operatorname{det} A \leq 2^{\frac{n}{2}}$ for any $A \in\{0,1\}^{n \times n}$ with at most $2 n$ ones. Ryser improved this estimate, and at the same time extended it to cover more general numbers of 1-entries in $A$ :

Theorem 2 (Ryser [18]). Let $A \in\{0,1\}^{n \times n}$ be a matrix containing exactly $k n$ ones. If $n \geq 2$ and $1 \leq k \leq \frac{n+1}{2}$ then, for $\lambda=k(k-1) /(n-1)$, it follows that

$$
|\operatorname{det} A| \leq k(k-\lambda)^{\frac{1}{2}(n-1)} \text {. }
$$


Moreover, Ryser showed that the bound is tight for many pairs of $k, n$; see next section. Inspecting Ryser's bound, we see that in the situation of Question 1, when $k \leq 2$, it yields a bound of $\operatorname{det} A \leq 2(2-o(1))^{\frac{n-1}{2}}$, which is not much better than the bound of $2^{\frac{n}{2}}$ that we get from Hadamard's inequality. Given that Ryser's bound is tight for many $k, n$, does that mean that we cannot hope for a better bound? No, it turns out. Our main result is:

Theorem 3. If $A \in\{0,1\}^{n \times n}$ has at most $2 n$ non-zero entries, then $|\operatorname{det}(A)| \leq 2^{n / 6} \cdot 3^{n / 6} \approx$ $1.348^{n}$.

While a substantial improvement on the bound of $2^{\frac{n}{2}} \approx 1.414^{n}$, the bound in Theorem 3 is almost certainly not best possible. Indeed, the best lower bound we have found comes from matrices of the kind

$$
A=\operatorname{diag}(C, \ldots, C), \text { where } C=\left(\begin{array}{lll}
1 & 1 & 0 \\
0 & 1 & 1 \\
1 & 0 & 1
\end{array}\right),
$$

that have determinant $2^{\frac{n}{3}} \approx 1.260^{n}$. We believe that larger determinants are impossible:

Conjecture 4. If $A \in\{0,1\}^{n \times n}$ has at most $2 n$ non-zero entries, then $|\operatorname{det}(A)| \leq 2^{n / 3}$.

We discuss the conjecture a bit more in the next section, and we prove Theorem 3 in Sections 3 and 4. As a tool in the proof we consider incidence matrices $I$ of graphs. Somewhat strikingly, we find that the determinant $\operatorname{det}\left(I I^{\top}\right)$ of the Gramian of the incidence matrix of a tree does not depend on the structure of the tree (Theorem 7 ).

We also consider two other types of combinatorial matrices. Many 0/1-matrices arise in combinatorial optimisation as constraints matrices of (mixed) integer programs. A particularly nice type of such a matrix are those with the consecutive ones property. These are 0/1-matrices, in which in each row all the one entries are consecutive. It is a standard exercise to show that matrices with the consecutive ones property are totally unimodular, which means that solving the linear relaxation also solves the corresponding integer program.

We consider two types of generalisations of the consecutive ones property. In Section 5 , we treat matrices with the $k$-consecutive ones property: matrices in whose rows the 1-entries appear in at most $k$ blocks. We will prove an upper bound on the determinant of such matrices (Theorem 9). Perhaps not immediately recognisable as a generalisation are path-edge incidence matrices of a tree. We treat these in Section [6] and similarly prove an upper bound on the determinant (Theorem 12). Finally, in Section 7, we use the bound of Theorem 12 in order to upper-bound a somewhat complicated graph parameter, the leaf rank of a graph. Our result is the first that bounds the leaf rank in terms of the order of the graph.

\section{Discussion}

Ryser's inequality in Theorem 2 is tight in many circumstances. Ryser proved that equality holds if and only if $A$ is the incidence matrix of an $(n, k, \lambda)$-configuration, that is, when $A$ is the incidence matrix of a collection of $n$ subsets $S_{1}, \ldots, S_{n}$ of $[n]$ such that $\left|S_{i}\right|=k$ and $\left|S_{i} \cap S_{j}\right|=\lambda$ for every $i \in[n]$ and every $j \in[n] \backslash\{i\}$. (We write $[n]$ as a shorthand for $\{1, \ldots, n\}$.)

As a consequence, equality in Theorem 2 may only hold if $\lambda$ is an integer, which implies $\lambda \geq 1$. That, in turn, entails $k \geq \sqrt{n}$. To sum up, Ryser's theorem can only be tight if the matrix $A$ contains at least $n \sqrt{n}$ many ones. This explains why for only $2 n$ ones, or indeed any linear number of ones, improvements may be possible.

There is another well-known bound on the determinant of a 0/1-matrix that comes from a very elegant transformation that turns any matrix $A \in\{0,1\}^{n \times n}$ into a matrix $B \in\{-1,1\}^{(n+1) \times(n+1)}$ 
such that $2^{n} \operatorname{det} A=\operatorname{det} B$; see [5]. In particular, if $A$ is any 0/1-matrix (with possibly more than $2 n$ ones), then direct application of Hadamard's inequality (11) to $A$ yields $|\operatorname{det} A| \leq n^{\frac{n}{2}}$, while an application of the inequality to $B$ yields the better bound of $|\operatorname{det}(A)| \leq 2^{-n}(n+1)^{(n+1) / 2}$. In our setting, however, where $A$ contains at most $2 n$ ones, the latter bound is actually worse than what we can get from (11) by exploiting that, on average, every row of $A$ has Euclidean norm at most $\sqrt{2}$.

Conjectures need evidence. As a first piece of evidence for Conjecture 4 we quickly prove a special case, where, more strongly, we require that every row of the matrix contains at most two ones:

Proposition 5. If $A \in\{0,1\}^{n \times n}$ and if every row of $A$ contains at most two ones, then $|\operatorname{det}(A)| \leq 2^{n / 3}$.

Proof. Clearly, we may assume that $A$ is non-singular. The proof is by induction on $n$. For $n \leq 3$, the bound is easily verified. Now, let $n>4$. If some row or column of $A$ contains at most one non-zero entry, then expanding the determinant of $A$ along this row or column, we obtain, by induction, $|\operatorname{det}(A)| \leq 2^{(n-1) / 3}$. Hence, every row and column of $A$ contains exactly two non-zero entries. Since $A$ is non-singular, this implies that, suitably permuting the rows and columns of $A$, we may assume that

$$
A=\left(\begin{array}{cc}
A_{1} & 0 \\
0 & A_{2}
\end{array}\right)
$$

where $A_{1}$ is the vertex-edge incidence matrix of a cycle $C_{\ell}$. If $\ell$ is even, then $\operatorname{det}\left(A_{1}\right)=0$, and, if $\ell$ is odd, then $\left|\operatorname{det}\left(A_{1}\right)\right|=2$. Since $A$ is non-singular, we obtain that $\ell$ is an odd integer at least 3 . By induction, we obtain

$$
|\operatorname{det}(A)|=\left|\operatorname{det}\left(A_{1}\right)\right| \cdot\left|\operatorname{det}\left(A_{2}\right)\right| \leq 2 \cdot 2^{(n-\ell) / 3} \leq 2^{n / 3},
$$

which completes the proof.

Our main result is to improve on Ryser's theorem for matrices that contain up to $2 n$ ones. But what about matrices with more ones? What about matrices with up to $3 n$ ones? While we do not know how to prove a small bound on the determinant, we at least can offer a lower bound that arises in a similar way as for $2 n$ ones. For this, denote by $C \in\{0,1\}^{7 \times 7}$ the incidence matrix of the Fano plane, or a $(7,3,1)$-configuration in Ryser's terminology. Then $\operatorname{det} C=3 \cdot 2^{\frac{7-1}{2}}=24$, by Ryser's theorem (or elementary calculation). Thus the $n \times n$-matrix $A=\operatorname{diag}(C, \ldots, C)$ has determinant $24^{\frac{n}{7}} \approx 1.57^{n}$. We suspect that this is the maximal determinant for $3 n$ ones.

\section{Gramians of graphs}

In this section we prove the main lemma for the proof of Theorem 3

Lemma 6. Let $I \in\{0,1\}^{m \times n}$ be a matrix that contains exactly two ones in each row. Then

$$
\operatorname{det}\left(I I^{\top}\right) \leq \begin{cases}2^{m} & \text { if } m \leq \frac{n}{2} \text { and } \\ 2^{\frac{1}{3}(n+m)} & \text { if } m \geq \frac{n}{2} .\end{cases}
$$

We recall some basic properties about the Gramian $I I^{\top}$ that hold for any matrix $I \in \mathbb{R}^{m \times n}$. These properties follow quite easily from properties of determinants: If $J$ arises from $I$ by 
$\left(\mathrm{G}_{1}\right)$ either multiplying some row with -1 ,

$\left(\mathrm{G}_{2}\right)$ or adding a multiple of some row to another row,

$\left(\mathrm{G}_{3}\right)$ or permuting rows and columns,

then $\operatorname{det}\left(J J^{\top}\right)=\operatorname{det}\left(I I^{\top}\right)$. Consider, for instance, the situation that $J$ arises from $I$ by adding $\alpha I_{j}$. to $I_{i}$. for distinct indices $i$ and $j$, and some real $\alpha$. If $A=I I^{\top}$, and $B$ arises from $A$ by adding $\alpha A_{j, \bullet}$ to $A_{i, \bullet}$, then $J J^{\top}$ arises from $B$ by adding $\alpha B \bullet_{, j}$ to $B \boldsymbol{\bullet}_{, i}$. Since both operations do not change the determinant, $\left(\mathrm{G}_{2}\right)$ follows.

If $I \in\{0,1\}^{m \times n}$ is a $0 / 1$-matrix with exactly two ones in each row, so if $I$ is as in Lemma 6 , then in particular $I$ may be seen as the edge-vertex incidence matrix of a graph $G$ with vertex set $\left\{v_{1}, \ldots, v_{n}\right\}$ and edge set $\left\{e_{1}, \ldots, e_{m}\right\}$ such that $v_{i}$ is incident with $e_{j}$ precisely when $I_{j, i}=1$. We find this view quite convenient as many of the arguments and statements we will need are easier to phrase in the language of graphs. For all basic graph-theoretical definitions and concepts we refer to the textbook of Diestel 8 . We note that all our graphs will be finite, simple and undirected.

There is a long interest in the algebraic properties of various matrices associated with graphs. For instance, Mowshowitz 14 showed that the characteristic polynomial of the adjacency matrix of a tree can be expressed in terms of matchings. The matrix $I I^{\top}$, with $I$ being the incidence matrix of the graph $G$, is closely related to the Laplacian of a graph. Indeed, if $Q$ is the incidence matrix of an orientation of $G$, then the Laplacian of $G$ is defined as $L=Q^{\top} Q$. It is not hard to verify that $L$ does not depend on the actual orientation. We refer to the book of Chung [7] for more on the Laplacian and other topics in spectral graph theory. While the Laplacian has rows and columns that correspond to the vertices of $G$, the rows and columns of the matrix $I I^{\top}$ correspond to the edges of $G$. Thus, the matrix $I I^{\top}$ that we are interested in, is even more closely related to the edge-Laplacian, defined as $Q Q^{\top}$, of the graph. For some results about the edge-Laplacian see Bapat 4 .

Often the determinant of a matrix associated with a graph $G$ encodes properties of $G$. In contrast, we find that, for a tree, the determinant $\operatorname{det}\left(I I^{\top}\right)$ does not depend on the structure of $T$ at all.

Theorem 7. If $T$ is a tree of order $n \geq 2$, and $I \in\{0,1\}^{E(T) \times V(T)}$ is the edge-vertex incidence matrix of $T$, then $\operatorname{det}\left(I I^{\top}\right)=n$.

Proof. For a function $s: V(T) \rightarrow\{-1,1\}$, let $I_{s}$ arise from $I$ by multiplying the column with index $u$ by $s(u)$ for every vertex $u$ of $T$. We say that $s$ is special, if $s(u)=1$ for every non-leaf $u$ of $T$. Note that $I_{s} I_{s}^{\top}=I I^{\top}$ if $s$ is special. We prove the following slightly more general statement: If $T$ is a tree of order $n, s: V(T) \rightarrow\{-1,1\}$ is special, and $I$ is the edge-vertex incidence matrix of $T$, then $\operatorname{det}\left(I_{s} I_{s}^{\top}\right)=n$.

First, if $T$ is a star, then

$$
\operatorname{det}\left(I_{s} I_{s}^{\top}\right)=\operatorname{det}\left(\begin{array}{cccccc}
2 & 1 & 1 & \ldots & 1 & 1 \\
1 & 2 & 1 & \ldots & 1 & 1 \\
1 & 1 & 2 & \ldots & 1 & 1 \\
\vdots & \vdots & \vdots & \ddots & \vdots & \vdots \\
1 & 1 & 1 & \ddots & 2 & 1 \\
1 & 1 & 1 & \ldots & 1 & 2
\end{array}\right)=\operatorname{det}\left(\begin{array}{cccccc}
2 & 1 & 1 & \ldots & 1 & 1 \\
-1 & 1 & 0 & \ldots & 0 & 0 \\
0 & -1 & 1 & \ldots & 0 & 0 \\
\vdots & \vdots & \vdots & \ddots & \vdots & \vdots \\
0 & 0 & 0 & \ddots & 1 & 0 \\
0 & 0 & 0 & \ldots & -1 & 1
\end{array}\right)=n,
$$

where the last equality follows easily by induction expanding the determinant along the final column. (Alternatively, the result also follows very quickly with the help of Sylvester's determinant identity.) 
Next, we consider a simple operation moving a leaf within a tree. More precisely, let $T$ be such that $u v w$ is a path in $T$, where $u$ is a leaf, and $w$ is not a leaf. Let $T^{\prime}=T-u v+u w$, and let

$$
s^{\prime}: V\left(T^{\prime}\right) \rightarrow\{-1,1\}: x \mapsto \begin{cases}-s(x) & , \text { if } x=u, \text { and } \\ s(x) & , \text { if } x \neq u .\end{cases}
$$

Clearly, since $s$ is special, the function $s^{\prime}$ is special. Furthermore, if $I^{\prime}$ is the edge-vertex incidence matrix of $T^{\prime}$, then $I_{s^{\prime}}^{\prime}$ arises from $I_{s}$ by subtracting the row with index $v w$ from the row with index $u v$, and multiplying the new row with index $u v$ with -1 , which, by $\left(\mathrm{G}_{1}\right)$ and $\left(\mathrm{G}_{2}\right)$, implies $\operatorname{det}\left(I^{\prime}{ }_{s^{\prime}} I_{s^{\prime}}^{\prime \top}\right)=\operatorname{det}\left(I_{s} I_{s}^{\top}\right)$.

Since every tree can be transformed to a star by a sequence of such simple operations, the desired statement follows.

Let $G$ be a connected graph of order $n$ and size $m$, and let $I$ be the edge-vertex incidence matrix of $G$. If $m>n$, then the rows of $I$ are linearly dependent, which, by $\left(\mathrm{G}_{2}\right)$, easily implies $\operatorname{det}\left(I I^{\top}\right)=0$. If $m=n$, then $G$ arises by attaching (possibly trivial) trees to the vertices of some cycle $C$. Iteratively expanding the determinant of the square matrix $I$ along columns corresponding to vertices of degree 1 , we obtain that $\operatorname{det}(I)=\operatorname{det}(J)$, where $J$ is the edge-vertex incidence matrix of $C$. It is a simple known fact that, if $C$ has even order, then $\operatorname{det}(J)=0$, and, if $C$ has odd order, then $|\operatorname{det}(J)|=2$.

Altogether, we obtain

$$
\operatorname{det}\left(I I^{\boldsymbol{\top}}\right)= \begin{cases}n & \text { if } G \text { is a tree, } \\ 0 & \text { if } m>n \text { or } G \text { contains an even cycle, and } \\ 4 & \text { if } m=n \text { and } G \text { contains an odd cycle. }\end{cases}
$$

Our next result is Lemma 6 phrased in terms of graphs.

Lemma 6b If $G$ is a graph of order $n$ that has at least one and at most $m$ edges, and $I$ is the edge-vertex incidence matrix of $G$, then

$$
\operatorname{det}\left(I I^{\top}\right) \leq \begin{cases}2^{m} & \text { if } m \leq \frac{n}{2} \text { and } \\ 2^{\frac{1}{3}(n+m)} & \text { if } m \geq \frac{n}{2}\end{cases}
$$

Proof. Among all graphs with $n$ vertices and at most $m$ edges (but at least one), let $G$ be chosen in such a way that $\operatorname{det}\left(I I^{\top}\right)$ is largest possible, and, subject to this condition, the number of edges of $G$ is smallest possible. Clearly $\operatorname{det}\left(I I^{\top}\right)>0$. Let $G$ have $k$ components $G_{1}, G_{2}, \ldots, G_{k}$ with edge-vertex incidence matrices $I_{1}, I_{2}, \ldots, I_{k}$. Since, by $\left(\mathrm{G}_{3}\right)$, permuting rows and columns does not affect $\operatorname{det}\left(I I^{\top}\right)$, we may assume that $I I^{\top}$ is a block diagonal matrix with $k$ blocks each corresponding to one component. Then

$$
\operatorname{det}\left(I I^{\boldsymbol{\top}}\right)=\operatorname{det}\left(I_{1} I_{1}^{\boldsymbol{\top}}\right) \cdot \operatorname{det}\left(I_{2} I_{2}^{\boldsymbol{\top}}\right) \cdots \operatorname{det}\left(I_{k} I_{k}^{\boldsymbol{\top}}\right) .
$$

We claim that

(i) every non-tree component of $G$ is a triangle, and

(ii) every tree component of $G$ is either an isolated vertex, or an edge, or a path on three vertices. 
Indeed, let $J$ be the edge-vertex incidence matrix of a triangle. Since, again by (2), $\operatorname{det}\left(I_{i} I_{i}^{\top}\right)$ equals $\operatorname{det}\left(J J^{\top}\right)$ if $G_{i}$ is a non-tree component, and since $G$ has as few edges as possible, we obtain (i). Suppose that $G$ has a tree component of at least $r \geq 4$ vertices. Then replacing the component by two components, one a tree on $r-2$ vertices and the other an edge, results, by (2), in an overall larger determinant. This proves (ii).

For $i \in[3]$, let $k_{i}$ be the number of components of $G$ with exactly $i$ edges. It follows that

$$
\operatorname{det}\left(I I^{\top}\right)=2^{k_{1}} \cdot 3^{k_{2}} \cdot 4^{k_{3}}=2^{k_{1}+\log _{2}(3) k_{2}+2 k_{3}},
$$

and that the non-negative integers $k_{1}, k_{2}$, and $k_{3}$ satisfy

$$
\begin{aligned}
k_{1}+2 k_{2}+3 k_{3} & \leq m \text { and } \\
2 k_{1}+3 k_{2}+3 k_{3} & \leq n .
\end{aligned}
$$

Since $\log _{2}(3) \leq \frac{5}{3} \leq 2$, we obtain

$$
\begin{aligned}
\log _{2}\left(\operatorname{det}\left(I I^{\top}\right)\right) & \stackrel{\sqrt[3]{\leq}}{\leq} k_{1}+2 k_{2}+3 k_{3} \stackrel{\text { 4 }}{\leq} m, \text { and } \\
\log _{2}\left(\operatorname{det}\left(I I^{\top}\right)\right) & \stackrel{\sqrt{3}}{\leq} k_{1}+\frac{5}{3} k_{2}+2 k_{3} \\
& =\frac{1}{3}\left(k_{1}+2 k_{2}+3 k_{3}\right)+\frac{1}{3}\left(2 k_{1}+3 k_{2}+3 k_{3}\right) \stackrel{\text { 4 , (5) }}{\leq} \frac{m+n}{3},
\end{aligned}
$$

which completes the proof.

Lemma 6b is tight. In fact, if $m \leq \frac{n}{2}$, then $G=m K_{2} \cup(n-2 m) K_{1}$ yields equality, and, if $\frac{n}{2} \leq m \leq n$ and $2 m-n$ is divisible by 3 , then $G=(n-m) K_{2} \cup \frac{1}{3}(2 m-n) K_{3}$ yields equality.

\section{Proof of Theorem 3}

For the proof of Theorem [3, we need another property of the Gramian. Let $B=\left(\begin{array}{l}A \\ a\end{array}\right)$ be a real matrix with last row $a$. If $a^{\prime}$ is the orthogonal projection of $a$ onto the row space of $A$, then, by $\left(\mathrm{G}_{2}\right)$,

$$
\begin{aligned}
\operatorname{det}\left(B B^{\boldsymbol{\top}}\right) & =\operatorname{det}\left(\left(\begin{array}{c}
A \\
a-a^{\prime}
\end{array}\right)\left(\begin{array}{c}
A \\
a-a^{\prime}
\end{array}\right)^{\top}\right)=\operatorname{det}\left(\begin{array}{cc}
A A^{\top} & 0 \\
0 & \left\|a-a^{\prime}\right\|_{2}^{2}
\end{array}\right) \\
& =\operatorname{det}\left(A A^{\top}\right) \cdot\left\|a-a^{\prime}\right\|_{2}^{2} \\
& \leq \operatorname{det}\left(A A^{\top}\right) \cdot\|a\|_{2}^{2} .
\end{aligned}
$$

Proof of Theorem [3. Clearly, we may assume that $A$ is non-singular; in particular, every row of $A$ contains at least one 1-entry.

Iteratively, expand the $\operatorname{determinant} \operatorname{det} A$ over rows with only one 1 -entry, and let $n_{1}$ be the number of rows eliminated in this way, and let $A^{\prime}$ be the resulting $\left(n-n_{1}\right) \times\left(n-n_{1}\right)$-matrix with $|\operatorname{det}(A)|=\left|\operatorname{det}\left(A^{\prime}\right)\right|$. In particular, $A^{\prime}$ contains at most $2 n-n_{1}$ many ones, and every row of $A^{\prime}$ contains at least two ones. Denote by $A_{2}$ the matrix consisting of the rows of $A^{\prime}$ with exactly two ones, and let $A_{3}$ be the matrix consisting of the other rows (each with at least three ones). Let $A_{i}$ have $n_{i}$ rows for $i=2,3$. Since $n_{1}+2 n_{2}+3 n_{3} \leq 2 n=2 n_{1}+2 n_{2}+2 n_{3}$, we obtain $n_{3} \geq n_{1}$. 
Iteratively applying (6), we obtain

$$
\operatorname{det}(A)^{2} \leq \operatorname{det}\left(A_{2} A_{2}^{\mathrm{\top}}\right) \cdot \prod_{i=1}^{n_{3}}\left\|A_{3,} \cdot\right\|_{2}^{2}
$$

Noting that $A_{2}$ has $n-n_{1}$ many columns and $n_{2}$ many rows, we apply Lemma 6 to get

$$
\operatorname{det}\left(A_{2} A_{2}^{\top}\right) \leq \begin{cases}2^{n_{2}} & \text { if } n_{2} \leq \frac{n-n_{1}}{2} \text { and } \\ 2^{\frac{1}{3}\left(\left(n-n_{1}\right)+n_{2}\right)} & \text { if } n_{2} \geq \frac{n-n_{1}}{2}\end{cases}
$$

Since $A_{3}$ is a $0 / 1$-matrix with at most $2 n-n_{1}-2 n_{2}=n_{1}+2 n_{3}$ non-zero entries, the inequality between the geometric and the arithmetic mean implies

$$
\prod_{i=1}^{n_{3}}\left\|A_{3, \cdot}\right\|_{2}^{2} \leq\left(\frac{1}{n_{3}} \sum_{i=1}^{n_{3}}\left\|A_{3,} \cdot\right\|_{2}^{2}\right)^{n_{3}} \leq\left(\frac{2 n-n_{1}-2 n_{2}}{n_{3}}\right)^{n_{3}}=\left(2+\frac{n_{1}}{n_{3}}\right)^{n_{3}} .
$$

Using $n=n_{1}+n_{2}+n_{3}$, we obtain

$$
\operatorname{det}(A)^{2} \leq \begin{cases}2^{n-n_{1}-n_{3}} \cdot\left(2+\frac{n_{1}}{n_{3}}\right)^{n_{3}} & \text { if } n_{1}+2 n_{3} \geq n, \text { and } \\ 2^{\frac{1}{3}\left(2 n-2 n_{1}-n_{3}\right)} \cdot\left(2+\frac{n_{1}}{n_{3}}\right)^{n_{3}} & \text { if } n_{1}+2 n_{3} \leq n .\end{cases}
$$

Setting $x=\frac{n_{1}}{n}$ and $y=\frac{n_{3}}{n}$, we rewrite the right-hand side as $2^{f(x, y) n}$, where

$$
f(x, y)= \begin{cases}1-x-y+\frac{1}{\ln (2)} \cdot y \cdot \ln \left(2+\frac{x}{y}\right) & \text { if } x+2 y \geq 1, \text { and } \\ \frac{1}{3}(2-2 x-y)+\frac{1}{\ln (2)} \cdot y \cdot \ln \left(2+\frac{x}{y}\right) & \text { if } x+2 y \leq 1 .\end{cases}
$$

Since $n_{3} \geq n_{1}$, we have $0 \leq y \leq x \leq 1$.

Let

$$
\begin{aligned}
& M_{1}=\max \{f(x, y): 0 \leq y \leq x \leq 1 \text { and } x+2 y \geq 1\} \text { and } \\
& M_{2}=\max \{f(x, y): 0 \leq y \leq x \leq 1 \text { and } x+2 y \leq 1\}
\end{aligned}
$$

Taylor expansion around $z=1$ implies $\ln (2+z) \leq \ln (3)+\frac{z-1}{3}$ for $z \geq 0$, and, hence,

$$
\begin{aligned}
& M_{1} \leq \max \{\underbrace{1-x-y+\frac{y}{\ln (2)}\left(\ln (3)+\frac{\frac{x}{y}-1}{3}\right)}_{=: g_{1}(x, y)}: 0 \leq y \leq x \leq 1, x+2 y \geq 1\} \\
& M_{2} \leq \max \{\underbrace{\frac{1}{3}(2-2 x-y)+\frac{y}{\ln (2)}\left(\ln (3)+\frac{\frac{x}{y}-1}{3}\right)}_{=: g_{2}(x, y)}: 0 \leq y \leq x \leq 1, x+2 y \leq 1\} .
\end{aligned}
$$

Since $\frac{\partial}{\partial x} g_{1}(x, y)$ is a negative constant and $\frac{\partial}{\partial y} g_{2}(x, y)$ is a positive constant, we obtain

$$
\begin{aligned}
& M_{1} \leq \max \left\{g_{1}(\max \{y, 1-2 y\}, y): 0 \leq y \leq 1\right\}, \text { and } \\
& M_{2} \leq \max \left\{g_{2}\left(x, \min \left\{x, \frac{1-x}{2}\right\}\right): 0 \leq x \leq 1\right\}
\end{aligned}
$$


Since $\frac{\partial}{\partial y} g_{1}(y, y)$ is a negative constant and $\frac{\partial}{\partial y} g_{1}(1-2 y, y)$ is a positive constant, we obtain $M_{1} \leq g_{1}(1 / 3,1 / 3)=\frac{1}{3}+\frac{\ln (3)}{3 \ln (2)}$. Since $\frac{\partial}{\partial x} g_{2}(x, x)$ is a positive constant and $\frac{\partial}{\partial x} g_{2}\left(x, \frac{1-x}{2}\right)$ is a negative constant, we obtain $M_{2} \leq g_{2}(1 / 3,1 / 3)=\frac{1}{3}+\frac{\ln (3)}{3 \ln (2)}$. Altogether, we obtain $|\operatorname{det}(A)| \leq$ $2^{\left(\frac{1}{3}+\frac{\ln (3)}{3 \ln (2)}\right) \frac{n}{2}}=2^{n / 6} \cdot 3^{n / 6} \approx 1.348^{n}$.

Obviously, we do not believe the bound in the theorem to be best possible, and indeed there are some ways to improve the bound further. Since we can assume that every column with a 1 in some row of $A_{2}$ or of $A_{3}$ contains at least two ones (otherwise we may expand over the column), we see that for every row $a$ in $A_{2}$ or in $A_{3}$ there is some other row $b$ with scalar product $a b^{\top} \geq 1$. Then, however, one of the two rows $a, b$ can be replaced by a shorter row. In this way, it is possible to shorten at least half of the rows in $A_{3}$ somewhat, which will result in a tighter estimation in (7). A similar approach is used in the proof of Theorem 10 below. We have not worked out the details here, because it is clear that this effect will not be enough to achieve the bound in Conjecture 4

\section{The $k$-consecutive ones property}

A 0/1-matrix $A$ has the consecutive ones property if, in each row of $A$, the 1-entries are consecutive. That is, in each row the ones form a block. We generalise this property by allowing more blocks of ones. For a positive integer $k$, let us say that $A$ has the $k$-consecutive ones property if in every row of $A$ the 1 -entries form at most $k$ blocks. That means every row has the form

$$
0 \ldots 0 \underbrace{1 \ldots 1}_{1 \text { st block }} 0 \ldots 0 \underbrace{1 \ldots 1}_{\text {2nd block }} 0 \ldots 0 \ldots 0 \ldots 0 \underbrace{1 \ldots 1}_{\text {lth block }} 0 \ldots 0
$$

for some $\ell \leq k$. It is well known that matrices with the consecutive ones property are totally unimodular: every square submatrix has determinant $-1,0$, or 1 . While the determinant of a matrix with the $k$-consecutive ones property is no longer bounded by any constant, we will still find a bound that is substantially better than Hadamard's bound (10).

We begin with a simple consequence of Hadamard's inequality:

Proposition 8. If $A \in\{-1,0,1\}^{n \times n}$ has $t$ non-zero entries, then

$$
|\operatorname{det}(A)| \leq\left(\frac{t}{n}\right)^{n / 2}
$$

with equality if $\frac{t}{n}$ and $\frac{n^{2}}{t}$ are integers, and there is a Hadamard matrix of order $\frac{t}{n}$.

Proof. For $i \in[n]$, let $s_{i}$ be the sum of the squares of the entries in column $i$ of $A$. Note that $\sum_{i=1}^{n} s_{i}=t$. By Hadamard's inequality (1), and, since the geometric mean is at most the arithmetic mean, we obtain

$$
|\operatorname{det}(A)| \leq \prod_{i=1}^{n} \sqrt{s_{i}} \leq\left(\frac{1}{n} \sum_{i=1}^{n} s_{i}\right)^{n / 2}=\left(\frac{t}{n}\right)^{n / 2}
$$

If $k=\frac{t}{n}$ and $\frac{n^{2}}{t}$ are integers, and $H$ is a Hadamard matrix of order $k$, then the block diagonal matrix $A$ with $\frac{n^{2}}{t}$ copies of $H$ along the diagonal satisfies

$$
|\operatorname{det}(A)|=|\operatorname{det}(H)|^{n^{2} / t}=\left(\left(\frac{t}{n}\right)^{t / 2 n}\right)^{n^{2} / t}=\left(\frac{t}{n}\right)^{n / 2}
$$


which completes the proof.

It is conceivable that equality holds in (8) if and only if $\frac{t}{n}$ and $\frac{n^{2}}{t}$ are integers, and there is a Hadamard matrix of order $\frac{t}{n}$. Note that, unlike Ryser's theorem, which has a similar setting, Proposition 8 is tight for some $n \times n$ matrices with only $O(n)$ non-zero entries.

Theorem 9. If $A \in\{0,1\}^{n \times n}$ has the $k$-consecutive ones property, then $|\operatorname{det}(A)| \leq(2 k)^{n / 2}$. Furthermore, for every $k$ such that a Hadamard matrix of order $k$ exists, and every $n$ for which $n / k$ is an integer, there is a matrix $A$ with $|\operatorname{det}(A)|=k^{(n-k) / 2}$ that has the $k$-consecutive ones property.

Proof. Let $A \in\{0,1\}^{n \times n}$ have the $k$-consecutive ones property. Let the matrix $B \in\{-1,0,1\}^{n \times n}$ arise from $A$ by subtracting, for $i$ from $n-1$ down to 1 , column $i$ from column $i+1$. Since $A$ has the $k$-consecutive ones property, every row of $B$ contains at most $2 k$ non-zero entries that alternate betwee 1 and -1 , and Proposition 8 implies $|\operatorname{det}(A)|=|\operatorname{det}(B)| \leq(2 k)^{n / 2}$.

Now, let $k$ and $n$ be such that a Hadamard matrix $H$ of order $k$ exists, and $p=n / k$ is an integer. Clearly, we may assume that $H_{\bullet_{1},}$ is the all-1-vector.

There is a matrix $R \in\{-1,0,1\}^{k \times k}$ such that $R \cdot, k$ is the all-0-vector, and in every row of

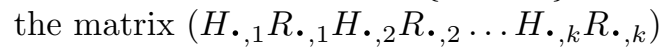

(i) there are at most $2 k$ non-zero entries,

(ii) the first non-zero entry is 1 , and

(iii) the 1-entries and -1 -entries alternate.

In fact, each column $R_{\bullet_{,} i}$ of $R$ with $i \leq k-1$ is uniquely determined by the columns $H_{\bullet_{, i}}$ and $H \cdot, i+1$ of $H$.

Let $\operatorname{Id}_{k}$ be the identity matrix of order $k$, and let the matrix $A$ of order $n=p k$ be as follows.

$$
A=\left(\begin{array}{cccccc}
H & R & 0 & \cdots & 0 & 0 \\
0 & H & R & \cdots & 0 & 0 \\
\vdots & \vdots & \ddots & \ddots & \vdots & \vdots \\
0 & 0 & 0 & \ddots & R & 0 \\
0 & 0 & 0 & \cdots & H & R \\
0 & 0 & 0 & \cdots & 0 & \mathrm{Id}_{k}
\end{array}\right)
$$

Clearly,

$$
|\operatorname{det}(A)|=|\operatorname{det}(H)|^{p-1}=k^{k(p-1) / 2}=k^{(n-k) / 2} .
$$

Let $v_{i}=A_{\bullet_{, i}}$ for $i \in[n]$, and let $B$ be the matrix equal to

$$
\left(v_{1} v_{k+1} \ldots v_{(p-1) k+1} v_{2} v_{k+2} \ldots v_{(p-1) k+2} \ldots v_{k-1} v_{k+(k-1)} \ldots v_{(p-1) k+(k-1)} v_{k} v_{2 k} \ldots v_{p k}\right) .
$$

Since $B$ arises by suitably permuting the columns of $A$, we obtain $|\operatorname{det}(B)|=|\operatorname{det}(A)|$, and the properties of $R$ imply that every row of $B$ satisfies (i)-(iii). Let $C$ arise from $B$ by adding, for $i$ from 1 up to $n-1$, column $i$ to column $i+1$.

The matrix $C$ has the $k$-consecutive ones property, and satisfies $|\operatorname{det}(C)|=k^{(n-k) / 2}$.

Unfortunately, there is a notable gap between the upper bound and the determinant of the matrix we construct. It is quite likely that that the upper bound can be improved substantially. The following result concerning the 2 -consecutive ones property illustrates possible ways of improving Theorem 9 , 
Theorem 10. If $A \in\{0,1\}^{n \times n}$ has the 2 -consecutive ones property, then $|\operatorname{det}(A)| \leq 3.936^{n / 2}$.

Proof. Let $A \in\{0,1\}^{n \times n}$ have the 2-consecutive ones property, and let $B \in\{-1,0,1\}^{n \times n}$ arise from $A$ exactly as in the beginning of the proof of Theorem 9 . Note that every row of $B$ contains at most four non-zero entries, and that, within every row that contains four non-zero entries, the -1-entries and the 1-entries alternate. Clearly, we may assume that the rows of $B$ are linearly independent, since otherwise $|\operatorname{det}(A)|=|\operatorname{det}(B)|=0$. Let $R$ be the set of rows of $B$ that contain four non-zero entries, let $S$ be the set of the remaining rows of $B$, and let $S^{\prime}=\emptyset$.

Our strategy to improve the bound from Theorem 9 exploits the fact that the rows in $R$ cannot all be pairwise orthogonal if $k=|R|$ is too big. More precisely, we will show that as long as $4 k>3 n$, there are two rows $a$ and $b$ in $R$ with $a b^{\top} \neq 0$. We remove $a$ from $R$, add the shorter row $a^{\prime}=a-\frac{a b^{\top}}{b b^{\top}} b$ to $S^{\prime}$, and iterate this process. Once $4 k \leq 3 n$, at least $n / 4$ rows are in $S \cup S^{\prime}$. Since a matrix whose set of rows is $R \cup S \cup S^{\prime}$ has the same determinant as $B$, an application of Hadamard's inequality (10) will yield the desired bound.

Therefore, let $4 k>3 n$. By the pigeon-hole principle, there are four rows $r_{1}, r_{2}, r_{3}$, and $r_{4}$ in $R$ that have a non-zero entry in the same column, say the column with index $j$. Suppose, for a contradiction, that $r_{i} r_{i^{\prime}}^{\top}=0$ for every two distinct indices $i$ and $i^{\prime}$ in [4]. Since the -1 -entries and the 1-entries alternate in each row in $R$, this implies that, for every two distinct indices $i$ and $i^{\prime}$ in [4], there are exactly two columns in which $r_{i}$ and $r_{i^{\prime}}$ both have non-zero entries. Furthermore, this latter property implies that there are no three rows among $r_{1}, r_{2}, r_{3}$, and $r_{4}$ that all have non-zero entries in the same two columns. Since multiplying rows by -1 affects neither the absolute value of the determinant nor the pairs of orthogonal rows, we may assume that the rows $r_{1}$ to $r_{4}$ have entry 1 in column $j$. Let $j_{2}, j_{3}$, and $j_{4}$ be the column indices distinct from $j$ in which row $r_{1}$ has its non-zero entries such that the entry in column $j_{3}$ is 1 . Since no three rows among $r_{1}, r_{2}, r_{3}$, and $r_{4}$ have non-zero entries in the same two columns, we may assume, by symmetry, that row $r_{2}$ has entry 1 in column $j_{2}$, row $r_{3}$ has entry -1 in column $j_{3}$, and row $r_{4}$ has entry 1 in column $j_{4}$. It follows that in the unique column distinct from columns $j, j_{2}$, and $j_{4}$, in which $r_{2}$ and $r_{4}$ both have a non-zero entry, both these rows have a -1-entry, which implies the contradiction that they cannot be orthogonal.

Altogether, it follows that for $4 k>3 n$, there are two columns $a$ and $b$ in $R$ that are not orthogonal. By symmetry, we may assume that $a b^{\top}>0$. Since the rows of $B$ are linearly independent, and since $a, b$ are integral, we obtain $a b^{\top} \geq 1$. Let $a^{\prime}=a-\frac{a b^{\top}}{b b^{\top}} b$. Then

$$
\left\|a^{\prime}\right\|_{2}^{2}=\|a\|_{2}^{2}-\frac{\left(a b^{\top}\right)^{2}}{\|b\|_{2}^{2}}
$$

which implies $\left\|a^{\prime}\right\|_{2} \leq \sqrt{15 / 4}$ as $\|a\|_{2}^{2}=4=\|b\|_{2}^{2}$.

Iterating the replacement of rows from $R$ by shorter rows in $S^{\prime}$ as long as $4 k>3 n$, we may thus assume that $R$ contains $k \leq 3 n / 4$ rows $r$ with $\|r\|_{2}=2$, and that $S \cup S^{\prime}$ contains $n-k \geq n / 4$ rows $r$ with $\|r\|_{2} \leq \sqrt{15 / 4}$. Since

$$
(\sqrt{15 / 4})^{n-k} 2^{k} \leq(\sqrt{15 / 4})^{n / 4} 2^{3 n / 4}=\left((\sqrt{15 / 4})^{1 / 2} 2^{3 / 2}\right)^{n / 2} \leq 3.936^{n / 2}
$$

Hadamard's inequality (11) implies the desired bound.

For the 2-consecutive ones property, we can also improve the lower bound of $2^{\frac{n-2}{2}} \approx 1.414^{n-2}$ from Theorem 9. More precisely, for every positive integer $n$ divisible by 3, there is an $n \times n$ 0/1-matrix $A$ that has the 2 -consecutive one property, and satisfies $\operatorname{det}(A)=4^{\frac{n-3}{3}} \approx 1.587^{n-3}$. 
The matrix $A$ is constructed as in the proof of Theorem 9 but with slightly different matrices $H$ and $R$ :

$$
H=\left(\begin{array}{ccc}
1 & 1 & -1 \\
1 & -1 & 1 \\
1 & -1 & -1
\end{array}\right) \text { and } R=\left(\begin{array}{ccc}
-1 & 0 & 0 \\
0 & 0 & 0 \\
0 & 1 & 0
\end{array}\right)
$$

We believe that this construction is essentially optimal:

Conjecture 11. If $A \in\{0,1\}^{n \times n}$ has the 2 -consecutive ones property, then $|\operatorname{det}(A)| \leq 4^{n / 3}$.

\section{Path-edge incidence matrices}

In the previous section we have generalised the consecutive ones property by allowing more blocks of ones. In this section we consider a different generalisation.

Let $P$ be a path with $m$ edges, and let $\mathcal{P}$ be a set of some $m$ subpaths of $P$. Then the path-edge incidence matrix $A(\mathcal{P}, P) \in\{0,1\}^{\mathcal{P} \times E(P)}$ defined as

$$
A(\mathcal{P}, P)_{Q, e}= \begin{cases}1 & \text { if } e \in E(Q), \text { and } \\ 0 & \text { otherwise }\end{cases}
$$

has the consecutive ones property (provided the edges are ordered as they appear in $P$ ). Conversely, any matrix with the consecutive ones property is the path-edge incidence matrix for suitable $\mathcal{P}$ and $P$. In this section we will consider path-edge incidence matrices $A(\mathcal{P}, T)$, where $\mathcal{P}$ is a set of subpaths in a tree $T$ rather than in a path.

We prove:

Theorem 12. If $T$ is a tree on $n$ vertices, and $\mathcal{P}$ is a set of $n-1$ paths in $T$, then

$$
|\operatorname{det}(A(\mathcal{P}, T))| \leq 2^{n-1}
$$

There is some relation between the two generalisations of the consecutive ones property. Trivially, if $T$ is the subdivision of a tree with $m$ edges, then $A(\mathcal{P}, T)$ has the $m$-consecutive ones property. Yet, not even all matrices with the 2-consecutive ones property can be represented in the form $A(\mathcal{P}, T)$. As an example, one may easily check that the matrix

$$
\left(\begin{array}{llll}
1 & 1 & 1 & 1 \\
1 & 1 & 1 & 0 \\
0 & 1 & 1 & 1 \\
1 & 0 & 0 & 1
\end{array}\right)
$$

has the 2-consecutive ones property but is not of the type $A(\mathcal{P}, T)$ for any tree $T$ and set of subpaths $\mathcal{P}$.

Proof of Theorem 12, Select a vertex $r$ in $T$ and consider $T$ as a rooted tree with root $r$. We denote by $\leq$ the tree order with minimal element $r$.

We turn the incidence matrix $A(\mathcal{P}, T)$ into a matrix $A$ by subtracting from every column with index $e$ all columns with index $e^{\prime}$ such that $e$ is the unique ancestor edge of $e^{\prime}$ in the tree order; this is done for all $e$ in breadth-first search order, that is, in non-decreasing tree order. Obviously, $A(\mathcal{P}, T)$ and $A$ have the same determinant.

For every $P \in \mathcal{P}$, the non-zero entries of row $A_{P,}$. fall into one of four cases: If $e, e^{\prime}$ are the two leaf-edges of $P$, then 
- if $e$ and $e^{\prime}$ are incomparable in the tree order and $P$ avoids $r$, then $A_{P,}$. has entry 1 at $e$ and at $e^{\prime}$, and entry -2 at the ancestor edge of the vertex of $P$ that is smallest in the tree order;

- if $e$ and $e^{\prime}$ are incomparable but $P$ passes through $r$, then $A_{P}$, has entry 1 at $e$ and at $e^{\prime}$ (and no entry -2 );

- if $e \leq e^{\prime}$ and $P$ avoids $r$, then the row has entry -1 at the ancestor edge of $e$ and entry 1 at $e^{\prime}$; and

- if $e \leq e^{\prime}$ and $P$ ends in $r$, then the row has a unique non-zero entry of 1 at $e^{\prime}$.

Note that at this point, Hadamard's inequality (11) already implies

$$
|\operatorname{det}(A(\mathcal{P}, T))| \leq 6^{\frac{n-1}{2}} .
$$

In order to obtain our better bound, we still need to work a bit.

Denote by $F$ the set of edges $e$ such that the column $A \bullet_{, e}$ has an entry -2 . For every $P \in \mathcal{P}$, the row $c=A_{P,}$. has the following properties:

(i) All edges $e$ with positive entries $c_{e}>0$ are incomparable in the tree order.

(ii) $c$ has at most one negative entry, and if $c_{e}=-2$, then $e \in F$.

(iii) If $c$ is negative at edge $e$ and positive at edge $f$, then $e \leq f$.

(iv) If $c_{e} \neq-2$, then $\left|c_{e}\right| \leq 1$ for every edge $e$.

(v) The sum of all positive entries is at most 2, that is, $\sum_{e \in E(T): c_{e}>0} c_{e} \leq 2$.

We now manipulate the rows of $A$ without changing the determinant and without losing properties (i)-(v) for any row. Let $F=\left\{e_{1}, \ldots, e_{k}\right\}$ and let $i$ be smallest such that the column $A$. $_{, e_{i}}$ also contains a positive entry.

Suppose that $P$ is a row index with $A_{P, e_{i}}=-2$ and that $Q$ is a row index with $A_{Q, e_{i}}>0$. Set $\alpha=A_{Q, e_{i}}$ and observe that $\alpha \leq 1$ by (iv). We replace the row $A_{Q}$, by a new row $A_{Q}, \cdot+\frac{\alpha}{2} A_{P,}$. and denote the new matrix by $A^{\prime}$. Clearly, $\operatorname{det}\left(A^{\prime}\right)=\operatorname{det}(A)$.

We claim that if $A$ had properties (i)-(v), then also $A^{\prime}$ has these properties.

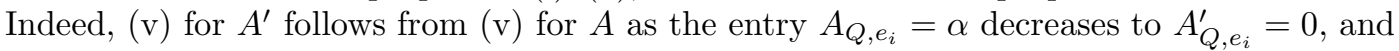

$$
\frac{\alpha}{2} \sum_{e \in E(T): A_{P, e}>0} A_{P, e} \leq \frac{\alpha}{2} \cdot 2=\alpha .
$$

In a similar way, (iii) for $A^{\prime}$ follows from (iii) for $A$ and $\alpha>0$.

If $A_{Q}$, has no negative entry, then (iii) holds trivially for $A_{Q}^{\prime}$. as $\alpha>0$. So, assume there is an edge $e$ with $A_{Q, e}<0$. It follows that $e$ is strictly smaller in the tree order than any $f$ with $A_{P, f}>0$ : By (iiii) for $A_{Q}$, , the edge $e$ is strictly smaller than $e_{i}$ as $A_{Q, e_{i}}>0$, and, by (iiii) for $A_{P,}$, the edge $e_{i}$ is smaller than any edge $f$ with $A_{P, f}>0$ as $A_{P, e_{i}}<0$. This ensures (iii) for $A^{\prime}$ as $A_{Q}^{\prime}$, can only have a negative entry at $e$.

Now, let $g$ be an edge of $P$ with $A_{P, g}>0$ and let $h \notin\left\{e_{i}, g\right\}$ be an edge of $Q$ with $A_{Q, h}>0$. Suppose that $g$ and $h$ are comparable. If $h \leq g$, then as also $e_{i} \leq g$, it follows that $h$ and $e_{i}$ are comparable, which is impossible by (ii) for $A_{Q}, \cdot$ If, on the other hand, $g \leq h$, then $e_{i} \leq g$ implies $e_{i} \leq h$, which is again impossible. Thus, $g$ and $h$ are incomparable. This guarantees (i) 
for $A^{\prime}$. Since $\frac{\alpha}{2} \leq 1$, it also guarantees (iv) as it implies that no two positive entries are added when forming the new row $A_{Q, \cdot}^{\prime}$.

Note that $A$ and $A^{\prime}$ have the same columns with -2 entries, namely those with index in $F$, and that no column of $A^{\prime}$ with index in $\left\{e_{1}, \ldots, e_{i-1}\right\}$ contains a positive entry since this is also the case for $A$. Note, moreover, that $A^{\prime}$ has one fewer positive entry in column $A_{\cdot, e_{i}}^{\prime}$, as $A_{Q, e_{i}}^{\prime}=0$. Thus, by replacing $A$ by $A^{\prime}$ and iteratively repeating this procedure, we can eliminate all positive entries in $A_{e_{,}}$. Doing this for all $i$ results in a matrix $B$ that satisfies (i)-(v), for which $\operatorname{det}(B)=\operatorname{det}(A)=\operatorname{det}(A(\mathcal{P}, T))$ and for which

$$
\text { no column with index in F contains a positive entry. }
$$

Define for such matrices $B$ two sets of row indices:

- $\mathcal{R}(B)$, the set of those $P \in \mathcal{P}$ for which an edge $e$ exists such that the column $B$, $e_{\text {, has a }}$ -2 at $P$ and zeros everywhere else; and

- $\mathcal{S}(B)$, the set of those $P \in \mathcal{P}$ that are 0 restricted to $F$ and for which $\left\|B_{P,} \cdot\right\|_{2} \leq 2$.

As long as there is some $P \in \mathcal{P} \backslash(\mathcal{R}(B) \cup \mathcal{S}(B))$ such that the row $B_{P}$. has an entry of -2 , we will iteratively modify $B$ so that the determinant does not change, properties (i) $-(\mathrm{v})$ are maintained for every row with index not in $\mathcal{R}(B) \cup \mathcal{S}(B)$, and (9) is maintained in such a way that $\mathcal{R}(B) \cup \mathcal{S}(B)$ grows in each step.

Assume that there is $P \in \mathcal{P} \backslash(\mathcal{R}(B) \cup \mathcal{S}(B))$ such that the row $B_{P,}$. has an entry of -2 at the edge $e$, say. By (ii), $e \in F$. Since $P \notin \mathcal{R}(B)$, there is a second non-zero entry in the column $B$.,$e$; let this be in row $Q \in \mathcal{P}$. By (9), we have $B_{Q, e}<0$. By the definition of $\mathcal{R}(B)$ and $\mathcal{S}(B)$, it follows that $Q \notin \mathcal{R}(B) \cup \mathcal{S}(B)$. In particular, (i)-(v) hold for the row $B_{Q}, \cdot$ Set $\beta=B_{Q, e}$ and replace the row $B_{Q}, \cdot$ by $B_{Q, \cdot}+\frac{\beta}{2} B_{P, \cdot}$ This results in a matrix $B^{\prime}$ of the same determinant as $B$. By (iii) for the rows $B_{P,}$, and $B_{Q, \cdot}$, and by (9), the new row $B_{Q,}^{\prime}$, is 0 in $F$, which means that $B^{\prime}$ satisfies (9).

By (iv) for $B_{Q, \cdot}$, we have $\beta \in[-2,0)$, which implies, together with (iv) and (ii) applied to $P$ and $Q$, that every entry of $B_{Q,}^{\prime}$. has absolute value at most 1 , that is, $\left\|B_{Q}^{\prime},\right\|_{\infty} \leq 1$. Moreover, from (v) we get

$$
\left\|B_{Q, \cdot}^{\prime}\right\|_{1} \leq \sum_{f: B_{Q, f}>0} B_{Q, f}+\frac{|\beta|}{2} \sum_{f: B_{P, f}>0} B_{P, f} \leq 2+1 \cdot 2=4 .
$$

This implies

$$
\left\|B_{Q, \cdot}^{\prime}\right\|_{2} \leq \sqrt{\left\|B_{Q, \cdot}^{\prime}\right\|_{1} \cdot\left\|B_{Q, \cdot}^{\prime}\right\|_{\infty}} \leq \sqrt{4 \cdot 1}=2,
$$

which means that $Q \in \mathcal{S}\left(B^{\prime}\right)$. Since the new row $B_{Q}^{\prime}$, is 0 in $F$, we have $\mathcal{R}(B) \subseteq \mathcal{R}\left(B^{\prime}\right)$. As also $\mathcal{S}(B) \subseteq \mathcal{S}\left(B^{\prime}\right)$, it follows that $\mathcal{R}\left(B^{\prime}\right) \cup \mathcal{S}\left(B^{\prime}\right)$ strictly includes $\mathcal{R}(B) \cup \mathcal{S}(B)$.

As the rows of $B^{\prime}$ with index outside of $\mathcal{R}\left(B^{\prime}\right) \cup \mathcal{S}\left(B^{\prime}\right)$ are the same as in $B$, it is clear that such rows still satisfy (i)-(v).

Let $C$ be the matrix obtained by a maximal sequence of such modifications, that is, for every $P \in \mathcal{P} \backslash(\mathcal{R}(C) \cup \mathcal{S}(C))$, the row $C_{P,}$. has no entry -2 . If $P \in \mathcal{P} \backslash(\mathcal{R}(C) \cup \mathcal{S}(C))$, then (iv) holds for $P$, and, hence, $\left\|C_{P,} \cdot\right\|_{\infty} \leq 1$. Now,

$$
\left\|C_{P, \cdot}\right\|_{2} \leq \sqrt{1 \cdot\left\|C_{P, \cdot}\right\|_{1}} \stackrel{\text { (iii), (iv) }}{\leq} \sqrt{1+\sum_{f: C_{P, f}>0} C_{P, f}} \leq \sqrt{3},
$$


where the last inequality follows from ( $(\mathbb{V})$ for $P$. As a consequence, we always get $\left\|C_{P} \cdot\right\|_{2} \leq 2$ for every $P \in \mathcal{P} \backslash \mathcal{R}(C)$. Note that the restriction of $C$ to rows in $\mathcal{R}(C)$ and columns in $F$ is, after permuting rows and columns, a diagonal matrix with -2 -entries in the diagonal. Thus, expanding the determinant of $C$ along the columns with index in $F$, followed by an application of Hadamard's inequality (11), results in $|\operatorname{det}(A(\mathcal{P}, T))|=|\operatorname{det}(C)| \leq 2^{n-1}$.

The bound can be improved by a factor of 4 by choosing the root as a leaf in which at least two paths from $\mathcal{P}$ end, which results in two unit vectors in $A(\mathcal{P}, T)$ along which one can expand the determinant.

We close this section with the construction of a tree $T$ of arbitrarily large order $n$, and a set $\mathcal{P}$ of $n-1$ paths in $T$ such that $|\operatorname{det}(A(\mathcal{P}, T))|=2^{\frac{2}{3} n-\frac{5}{3}}$, which limits the possible improvements of Theorem 12,

For any integer $n^{\prime}$, let $n \geq n^{\prime}$ such that there is a tree $T$ on $n$ vertices in which all internal vertices have degree 3 , and in which there is a vertex $r$ that has the same distance, $d$ say, to every leaf. Note that $T$ has $n-1=3\left(2^{d}-1\right)$ edges, and consider $T$ as rooted in $r$.

The set $\mathcal{P}$ of $m$ paths consists of two subsets, $\mathcal{P}_{I}$ and $\mathcal{P}_{L}$. For every edge $u v$ of $T$ between two non-leaves $u$ and $v$ of $T$ such that $u$ is the ancestor of $v$, select a leaf-leaf path $P_{u v}$ containing $v$ but not $u$; we denote the set of these paths by $\mathcal{P}_{I}$. Note that $\left|\mathcal{P}_{I}\right|=3\left(2^{d-1}-1\right)=\frac{n}{2}-2$. For the set $\mathcal{P}_{L}$ partition the leaves of $T$ into triples $\{a, b, c\}$ such that $a, b$, and $c$ are pairwise separated by the root $r$. For each such triple add the $a-b$ path $Q_{a, b}$, the $b-c$ path $Q_{b, c}$, and the $c-a$ path $Q_{c, a}$ to $\mathcal{P}_{L}$. Note that $\left|\mathcal{P}_{L}\right|=3 \cdot 2^{d-1}=\frac{n}{2}+1$.

As in the proof of the theorem, we turn the incidence matrix $A(\mathcal{P}, T)$ into a matrix $A$ by subtracting from each column the columns of direct descendant edges, where we go through the columns/edges in breadth-first search order. Any row of $A$ belonging to a path $P_{u v}$ in $\mathcal{P}_{I}$ has two +1 -entries at leaf-edges, a -2 -entry at $u v$, and 0 -entries everywhere else. Any row belonging to a path $Q_{a, b}$ in $\mathcal{P}_{L}$ has a +1 -entry at the leaf-edge incident with $a$, a +1 -entry at the leaf-edge incident with $b$, and 0-entries everywhere else. In particular, up to a permutation of its rows and columns, the matrix $A$ has the following form:

$$
\left(\begin{array}{cccccc}
-2 & \ldots & 0 & & & \\
\vdots & \ddots & \vdots & & * & \\
0 & \ldots & -2 & & & \\
& & & C & \ldots & 0 \\
& 0 & & \vdots & \ddots & \vdots \\
& & & 0 & \ldots & C
\end{array}\right) \text {, where } C=\left(\begin{array}{ccc}
1 & 1 & 0 \\
0 & 1 & 1 \\
1 & 0 & 1
\end{array}\right) .
$$

Since $\operatorname{det}(C)=2$, we obtain $|\operatorname{det}(A(\mathcal{P}, T))|=2^{\left|\mathcal{P}_{I}\right|} \cdot 2^{\frac{1}{3}\left|\mathcal{P}_{L}\right|}=2^{\frac{2}{3} n-\frac{5}{3}}$.

\section{An upper bound on the leaf rank}

Let us give an application of Theorem 12 .

There are various ways to capture a graph, or at least its essential properties, in terms of a tree. This often helps to understand the graph, since trees have a particularly simple structure. While tree decompositions are certainly the most prominent example, other approaches have been pursued as well. In the context of phylogenetic trees, for instance, Nishimura et al. 15] proposed the concept of leaf roots: Given a graph $G$ with vertex set $V(G)$ a tree $T$ is a $k$-leaf root of $G$, for some integer $k$, if

- the set of leaves of $T$ equals $V(G)$, and, 
- any two distinct vertices $u$ and $v$ of $G$ are adjacent in $G$ if and only if $\operatorname{dist}_{T}(u, v) \leq k$, that is, if their distance in $T$ is at most $k$.

Not all graphs have $k$-leaf roots for any given $k$, or indeed any $k$ at all. Brandstädt et al. 1 define the leaf rank of a graph $G$ : if there is an integer $k$ such that $G$ has a $k$-leaf root then let the leaf rank of $G$ be the smallest such $k$. If for no $k$ the graph $G$ has a $k$-leaf root then we say that $G$ has infinite leaf rank. The 4-cycle, for instance, has infinite leaf rank.

The structure of graphs with leaf rank at most 4 is well understood 2, 3, 9, 16. For leaf rank 5 at least an efficient recognition algorithm has been described [6], but for larger leaf rank both a structural characterisation and efficient recognition algorithms are unknown.

Even simpler properties are open, such as: How large, in terms of the order, can the leaf rank be (provided it is finite)? While Brandstädt et al. [1 prove that ptolemaic and interval graphs of order $n$ have leaf rank at most $2 n$, for general graphs an upper bound in terms of $n$ on the leaf rank was previously known. We prove such a bound:

Theorem 13. Every graph of order $n$ has either infinite leaf rank or leaf rank at most $2 n 2^{2 n}$.

Is the bound tight? On the contrary, it appears incredibly loose: We do not know of any graph on $n$ vertices that has finite leaf rank larger than $n$. Any polynomial bound in terms of $n$ would be very much welcome.

Proof. Let $G$ be a graph of order $n$, and assume $G$ to have finite leaf rank. For a tree $T$ whose set of leaves equals the vertex set $V(G)$ of $G$, we consider the following system $\mathcal{I}(T)$ of linear inequalities using the $n(T)$ real variables $\left(\ell_{e}\right)_{e \in E(T)}$ and $k$

$$
\mathcal{I}(T): \begin{cases}\ell(u T v)-k \leq 0 & \text { for every pair } u, v \text { of adjacent vertices of } G, \text { and } \\ \ell(u T v)-k \geq 1 & \text { for every pair } u, v \text { of non-adjacent vertices of } G\end{cases}
$$

where $u T v$ is the path in $T$ between the leaves $u$ and $v$ of $T$, and $\ell(P)=\sum_{e \in E(P)} \ell_{e}$ for any path $P$.

If $T$ is a $k$-leaf root of $G$, then $\left(\left(\ell_{e}\right)_{e \in E(T)}, k\right)$ with $\ell_{e}=1$ for $e \in E(T)$ is a non-negative integral solution of $\mathcal{I}(T)$. That is, the polyhedron

$$
\mathrm{P}(T)=\left\{\left(\left(\ell_{e}\right)_{e \in E(T)}, k\right) \in \mathbb{R}_{\geq 0}^{n(T)}:\left(\left(\ell_{e}\right)_{e \in E(T)}, k\right) \text { satisfies } \mathcal{I}(T)\right\}
$$

is non-empty. Conversely, for any tree $T$ whose set of leaves equals $V(G)$

if $(\ell, k)$ is an integral point of $\mathrm{P}(T)$ then the leaf rank of $G$ is at most $k$.

Now, let $T$ be a tree of minimum order such that $\mathrm{P}(T)$ is non-empty. Clearly, the tree $T$ has no vertex of degree 2 , which implies that the number of its internal vertices is not larger than the number of its leaves. Consequently

$$
n(T) \leq 2 n
$$

Suppose that $\mathrm{P}(T)$ contains a point $\left(\left(\ell_{e}\right)_{e \in E(T)}, k\right)$ that has a zero entry. As $k=0$ is impossible there is then some edge $e$ of $T$ with $\ell_{e}=0$. Contracting the edge $e$ within $T$ yields a smaller tree $T / e$ such that $\mathrm{P}(T / e)$ is non-empty, which contradicts the choice of $T$. Therefore, every point of $\mathrm{P}(T)$ has only strictly positive entries. By the convexity of polyhedra, this implies that the non-negativity condition is redundant, that is,

$$
\mathrm{P}(T)=\left\{\left(\left(\ell_{e}\right)_{e \in E(T)}, k\right) \in \mathbb{R}^{n(T)}:\left(\left(\ell_{e}\right)_{e \in E(T)}, k\right) \text { satisfies } \mathcal{I}(T)\right\}
$$


Since $\mathrm{P}(T)$ is contained in the positive orthant $\mathbb{R}_{>0}^{n(T)}$ and as it is non-empty, the polyhedron $\mathrm{P}(T)$ must have a vertex $x$. By a theorem of Hoffman and Kruskal [12, there is a subsystem $\mathcal{I}^{\prime}(T)$ of $\mathcal{I}(T)$ such that $x$ is the unique solution of the system of linear equalities obtained by turning the inequalities in $\mathcal{I}^{\prime}(T)$ into equalities. Since the ambient space is $n(T)$-dimensional, we may assume $\left|\mathcal{I}^{\prime}(T)\right|=n(T)$.

In view of the structure of $\mathcal{I}(T)$, there are two sets $\mathcal{P}_{0}$ and $\mathcal{P}_{1}$ of altogether $n(T)$ paths in $T$ such that

$$
\{x\}=\left\{\left(\left(\ell_{e}\right)_{e \in E(T)}, k\right) \in \mathbb{R}^{n(T)}: \ell(P)-k=i \text { for every } i \in\{0,1\} \text { and every } P \in \mathcal{P}_{i}\right\} .
$$

Let us write that in a slightly more concise way. Set $\mathcal{P}=\mathcal{P}_{0} \cup \mathcal{P}_{1}$ and $-\mathbf{1}=(-1, \ldots,-1)^{\top} \in$ $\mathbb{R}^{n(T)}$. Then there is a $0 / 1$-vector $\mathbf{b}$ of length $n(T)$ such that $x$ is the unique solution of the system $(A(\mathcal{P}, T),-\mathbf{1}) x=\mathbf{b}$ of linear equalities.

Denote by $A_{i}$ the matrix obtained from $(A(\mathcal{P}, T),-\mathbf{1})$ be replacing the $i$ th column by $\mathbf{b}$. Then, by Cramer's rule,

$$
x_{i}=\frac{\operatorname{det} A_{i}}{\operatorname{det}((A(\mathcal{P}, T),-\mathbf{1}))} .
$$

In particular, the last entry $k$ of $x$ equals

$$
k=\frac{\operatorname{det}((A(\mathcal{P}, T), \mathbf{b}))}{\operatorname{det}((A(\mathcal{P}, T),-\mathbf{1}))} .
$$

Thus, $|\operatorname{det}((A(\mathcal{P}, T),-\mathbf{1}))| x$ is an integral point of $\mathrm{P}(T)$. Recalling (10), we therefore see that

$$
k^{\prime}:=|\operatorname{det}((A(\mathcal{P}, T),-\mathbf{1}))| k=|\operatorname{det}((A(\mathcal{P}, T), \mathbf{b}))|
$$

is an upper bound on the leaf rank of $G$. Expanding the determinant of the matrix $(A(\mathcal{P}, T), \mathbf{b})$ along the last column $\mathbf{b}$, we obtain, by Theorem 12 and (11), that

$$
k^{\prime}=|\operatorname{det}((A(\mathcal{P}, T), \mathbf{b}))| \leq n(T) 2^{n(T)-1} \leq(2 n) 2^{2 n} .
$$

\section{References}

[1] A. Brandstädt, C. Hundt, F. Mancini, P. Wagner, Rooted directed path graphs are leaf powers, Discrete Mathematics 310 (2010), 897-910.

[2] A. Brandstädt, V.B. Le, Structure and linear time recognition of 3-leaf powers, Information Processing Letters 98 (2006), 133-138.

[3] A. Brandstädt, V.B. Le, D. Rautenbach, Distance-hereditary 5-leaf powers, Discrete Mathematics 309 (2009), 3843-3852.

[4] R.B. Bapat, Graphs and Matrices, Springer (2010).

[5] J. Brenner, L. Cummings, The Hadamard maximum determinant problem, American Mathematical Monthly 79 (1972), 626-630.

[6] M.-S. Chang, M.-T. Ko, The 3-Steiner root problem, Lecture Notes in Computer Science 4769 (2007), 109-120. 
[7] F. Chung, Spectral Graph Theory, CBMS Regional Conference Series in Mathematics (1997).

[8] R. Diestel, Graph Theory (4th edition), Springer (2010).

[9] M. Dom, J. Guo, F. Hüffner, R. Niedermeier, Error compensation in leaf root problems, Algorithmica 44 (2006), 363-381.

[10] R.L. Graham, H.O. Pollack, On the addressing problem for loop switching, The Bell System Technical Journal 50 (1971), 2495-2519.

[11] J. Hadamard, Résolution d'une question relative aux déterminants, Bulletin des Sciences Mathématiques 2 (1893), 240-246.

[12] A.J. Hoffman, J.B. Kruskal, Integral boundary points of convex polyhedra, in: Linear Inequalities and Related Systems, Annals of Mathematical Study 38 (H.W. Kuhn, A.W. Tucker, eds.), Princeton University Press, Princeton 1956, pp. 223-246.

[13] B.D. McKay, On the spectral characterization of trees, Ars Combinatoria 3 (1979), 219-232.

[14] A. Mowshowitz, The characteristic polynomial of a graph, Journal of Combinatorial Theory, Series B 12 (1972), 177-193.

[15] N. Nishimura, P. Ragde, D.M. Thilikos, On graph powers for leaf labeled trees, Journal of Algorithms 42 (2002), 69-108.

[16] D. Rautenbach, Some remarks about leaf roots, Discrete Mathematics 306 (2006), 14561461.

[17] R.E.A.C. Paley, On orthogonal matrices, Journal of Mathematics and Physics 12 (1933), 311-320.

[18] H.J. Ryser, Maximal determinants in combinatorial investigations, Canadian Journal of Mathematics 8 (1956), 245-249.

Version November 29, 2017

Henning Bruhn <henning. bruhn@uni-ulm.de>

Dieter Rautenbach <dieter.rautenbach@uni-ulm.de>

Institut für Optimierung und Operations Research

Universität Ulm

Germany 